\title{
Novel Synthesis of Ureas: Application of $t$-Butylureas
}

\author{
Ai Ito, Hideaki Muratake, and Koichi Shudo* \\ Research Foundation Itsuu Laboratory; 2-28-10 Tamagawa, Setagaya-ku, Tokyo 158-0094, Japan. \\ Received September 28, 2009; accepted October 30, 2009; published online November 4, 2009
}

Otherwise inaccessible tropolonylureas were prepared by reaction of $t$-butylurea with appropriate amines, with elimination of $t$-butylamine. This method is also generally applicable for urea synthesis.

Key words urea; $t$-butylurea; isocyanate; tropolone; substitution

Urea is an important pharmacophore which is present in various pharmaceutical drugs and agrochemicals. For example, terguride ${ }^{1)}$ is a dopamine agonist, many sulfonylureas ${ }^{2,3)}$ are used as therapeutics for diabetes, sorafenib $b^{4,5)}$ is used to treat renal cell carcinoma, $N$-(3,4-dichlorophenyl)- $N^{\prime}, N^{\prime}-$ dimethylurea (diuron) $\left.{ }^{6}\right)$ is a herbicide, and $N$-(2-chloro-4pyridyl)- $N^{\prime}$-phenylurea (forchlorofenuron) ${ }^{7)}$ is a plant growth regulator. ${ }^{8)}$ Such compounds may be prepared by conventional methods, $\left.{ }^{9}, 10\right)$ i.e., reaction of an amine with an isocyanate (or in situ-formed isocyanate generated by Curtius rearrangement of acid azide), ${ }^{11-14)}$ reaction of an amine and a carbamate, ${ }^{15-18)}$ or reaction of an amine and phosgene or its equivalents. ${ }^{19-21)}$ The last method is convenient, but sometimes encounters difficulties when the amine or intermediate is reactive. In some cases, none of these methods can be easily applied.

We have been investigating the structural and medicinal chemistry of tropolone (2-hydroxy-2,4,6-cycloheptatrien-1one), ${ }^{22)}$ which is a nonbenzenoid aromatic compound having a flat seven-membered ring. The tropolone fragment can be used as a pharmacophore instead of benzoic acid, phenol or other heteroaromatic moieties, ${ }^{23)}$ and its derivatives, e.g., thujaplicins and colchicines, possess antibacterial, antifungal, antiviral, and antitumor properties. ${ }^{24,25)}$ Some tropolone derivatives show significant retinoid activity. ${ }^{23)}$ Therefore, we set out to synthesize various tropolonylureas from 5aminotropolone (1). ${ }^{26,27)}$ When we attempted to extend tropolone chemistry, we found that compounds such as $N, N^{\prime}$ di(5-tropolonyl)urea (2) were not readily accessible from $\mathbf{1}$, since we could not obtain 5-tropolonyl isocyanate by conventional methods (e.g., using phosgene equivalents). Fortunately, we found a method to prepare compound 2 (Chart 1), and this method can also be applied to general synthesis of urea compounds, as described below.

\section{Results and Discussion}

5-Aminotropolone (1) reacts with various isocyanates, such as phenyl, substituted phenyl, and aliphatic isocyanates, leaving the hydroxy group intact. However, when 1 was treated with 1 eq of $t$-butyl isocyanate at reflux for $51 \mathrm{~h}$ in tetrahydrofuran (THF), $N, N^{\prime}$-di(5-tropolonyl)urea (2) was unexpectedly obtained as a major product instead of $\mathrm{N}-\mathrm{t}$ butyl- $N^{\prime}$-(5-tropolonyl)urea (3). In order to obtain 3, an excess amount of $t$-butyl isocyanate and a shorter reaction time are required, suggesting that $\mathbf{3}$ may serve as an intermediate in the formation of $\mathbf{2}$. Indeed, when isolated $\mathbf{3}$ was treated with 1 in toluene at $120^{\circ} \mathrm{C}$ for $14 \mathrm{~h}, 2$ was smoothly generated in a good yield of $82 \%$ (Chart 1 ).

When $N$-adamantyl- $N^{\prime}$-tropolonylurea was prepared and reacted with $\mathbf{1}$ under similar conditions, $\mathbf{2}$ was obtained in a lower yield of $36 \%$. The main reason for this may be that the adamantylamine is not readily removed during the reaction, because of its high boiling point. Therefore, the $t$-butylamine may be the best leaving alkylamine in terms of both bulkiness (high eliminability) and high volatility. This method for urea preparation, utilizing $t$-butylurea, should be available not only for 5-tropolonyureas, but also for general preparation of ureas (vide infra).

Reaction of $\mathbf{3}$ with a variety of primary and secondary amines (e.g., aromatic, heterocyclic, aliphatic amines) in toluene at $120^{\circ} \mathrm{C}$ afforded the desired ureas in good yield (Table 1, entries 1-19). A hindered amine, 2,6-dimethylaniline, reacted with 3 to give $4 a$ (entry 1 ). The reaction proceeded well with amines that are nucleophilic (e.g., entries 2 , $4,6)$ or weakly nucleophilic (e.g., entries $3,5,8,9,11)$. As expected, 3 reacted with piperazine, a bidentate amine, to afford $N$-piperazinyl- $N^{\prime}$-(5-tropolonyl)urea (4I) in $72 \%$ yield, leaving one amino group intact (entry 12). Ureas $\mathbf{4 m - p}$ were also readily obtained by the reaction of $\mathbf{3}$ with other aliphatic secondary amines (entries 13-16). The method was also extended to more complex tropolonylureas (entries $18,19)$. The reaction of $\mathbf{3}$ with bioactive substances, such as histamine and pindolol, provided the corresponding ureas $\mathbf{4 r}$ and $\mathbf{4 s}$ derivatized from the aliphatic amines, respectively, in high yield. The reactions of $N$-t-butyl- $N^{\prime}$-phenylurea (5) and $N$-t-butyl- $N^{\prime}$-(3-pyridyl)urea (7) with amines are illustrated

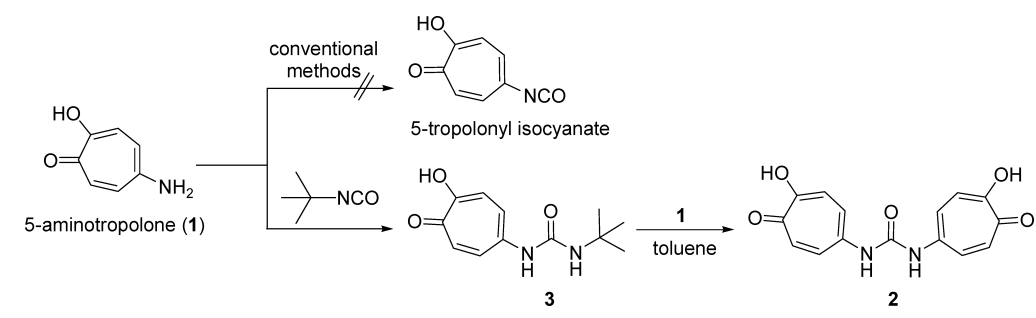

Chart 1 
Table 1. Reaction of $t$-Butylureas $(\mathbf{3}, \mathbf{5}, \mathbf{7})$ with Various Amines

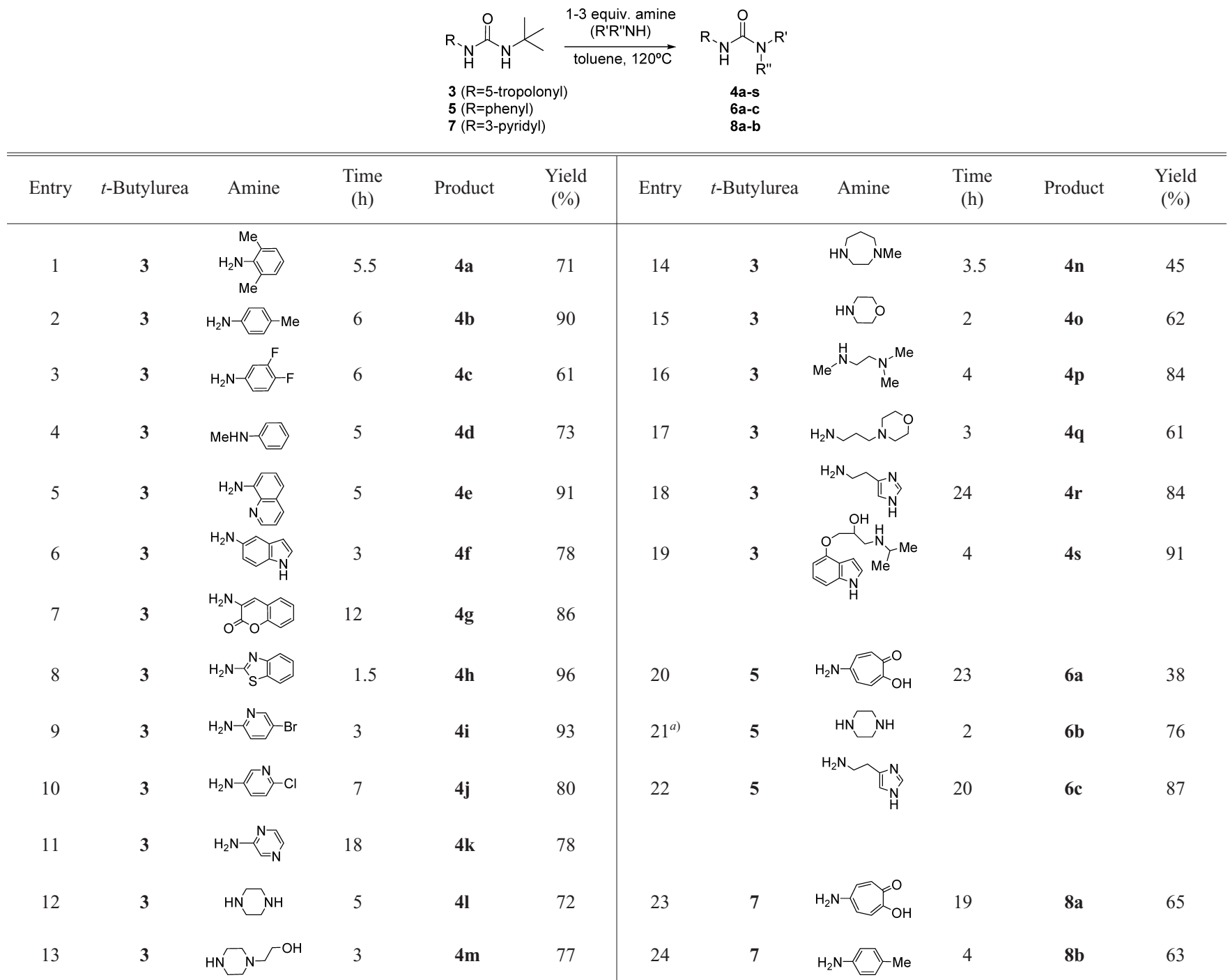

a) See Experimental.

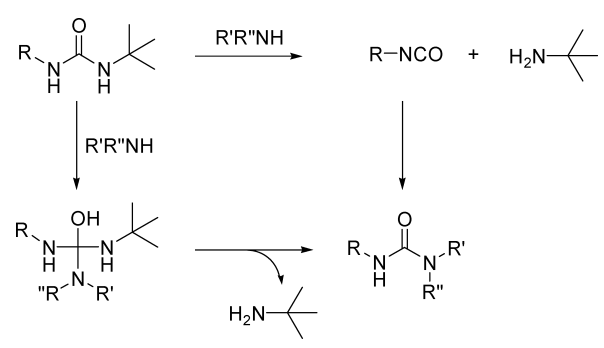

Chart 2

in entries $20-24$.

Possible reaction pathways include a stepwise reaction involving an isocyanate intermediate formed by elimination of $t$-butylamine, and/or direct nucleophilic substitution of the $t$ butylamine by amine $\left(\mathrm{R}^{\prime} \mathrm{R}^{\prime \prime} \mathrm{NH}\right)$ (Chart 2). It was reported that $N$-alkyl- $N^{\prime}$-phenylureas can be prepared from $N, N^{\prime}$ diphenylurea by treatment with aliphatic amines, though this reaction lacks versatility, and the reaction mechanism has not been established. ${ }^{28-30)}$ In the present reaction, the $t$-butylureas are stable at toluene reflux temperature, and simple thermal dissociation to the isocyanate intermediate is un- likely. ${ }^{31-34)}$ The presence of amine is necessary for the reaction to proceed. The amine may catalyze elimination of the $t$ butylamine to afford the isocyanate. Or, nucleophilic attack of the amine on the carbonyl group and subsequent removal of $t$-butylamine may occur. In any case, the formed $t$-butylamine (bp $44-46^{\circ} \mathrm{C}$ ) is highly volatile, and can be easily removed from the reaction medium. $t$-Butylamine evaporated from the reaction medium can be easily detected with $\mathrm{pH}$ test paper.

\section{Conclusion}

A simple and versatile method for the preparation of ureas, based on reaction of $t$-butylureas with amines, was developed. This method is especially useful for synthesizing urea compounds for which corresponding isocyanates or their precursors are not available.

\section{Experimental}

General Procedures Melting points were determined on a Yanagimoto micro-melting point apparatus (hot plate) without correction. Mass spectra were recorded on a Hitachi M-80B spectrometer at an ionizing voltage of $70 \mathrm{eV}$, and figures in parentheses indicate the relative intensities. IR spectra were measured on a Shimadzu IR-460 spectrophotometer. ${ }^{1} \mathrm{H}$ - and ${ }^{13} \mathrm{C}-\mathrm{NMR}$ 
spectra were obtained on a Varian Mercury 300 at $300 \mathrm{MHz}$ and at $75 \mathrm{MHz}$, respectively. Chemical shifts are recorded in ppm, and coupling constants $(J)$ in $\mathrm{Hz}$. Chemical shifts were calculated on the basis of tetramethylsilane (0 ppm for ${ }^{1} \mathrm{H}-\mathrm{NMR}$ in $\left.\mathrm{CDCl}_{3}\right),\left(\mathrm{C}_{3}\right)_{2} \mathrm{SO}\left(2.49 \mathrm{ppm}\right.$ for ${ }^{1} \mathrm{H}-\mathrm{NMR}$ in DMSO- $\left.d_{6}\right),\left(\underline{\mathrm{CH}}_{3}\right)_{2} \mathrm{SO}\left(39.5 \mathrm{ppm}\right.$ for ${ }^{13} \mathrm{C}-\mathrm{NMR}$ in DMSO- $\left.d_{6}\right)$, or $\mathrm{C}_{3} \mathrm{OH}$ (3.30 ppm for ${ }^{1} \mathrm{H}-\mathrm{NMR}$ in $\mathrm{CD}_{3} \mathrm{OD}$ ). Fuji Davison BW 300 or Merck aluminum oxide 90 was used for flash chromatography, and TLC was carried out on Merck Silica gel $60 \mathrm{PF}_{254}$

Reaction of 5-Aminotropolone (1) with 1 eq of $\boldsymbol{t}$-Butyl Isocyanate A solution of 5-aminotropolone $(\mathbf{1}, 206 \mathrm{mg}, 1.50 \mathrm{mmol})$ and $t$-butyl isocyanate $(171 \mu 1,1.50 \mathrm{mmol})$ in THF $(10 \mathrm{ml})$ was refluxed for $51 \mathrm{~h}$, then cooled to room temperature, and the solvent was evaporated in vacuo. The residue was washed with $\mathrm{MeOH}$ to give $N, N^{\prime}-\operatorname{di}(5$-tropolonyl)urea $(2,96 \mathrm{mg}, 43 \%)$ as dark brown powder, mp $258-260{ }^{\circ} \mathrm{C}$ (dec.). ${ }^{1} \mathrm{H}-\mathrm{NMR}$ (DMSO- $\left.d_{6}\right) \delta: 9.06$ $(2 \mathrm{H}$, br s, $\mathrm{NH} \times 2), 7.55(4 \mathrm{H}, \mathrm{d}, J=12.0 \mathrm{~Hz}), 7.21(4 \mathrm{H}, \mathrm{d}, J=12.0 \mathrm{~Hz}) . \mathrm{IR}$ $(\mathrm{KBr}) \mathrm{cm}^{-1}: 3195,1612,1548,1448,1419,1262$. MS m/z: $163\left(\mathrm{M}^{+}-137\right.$, 67), 137 (65), 135 (59), 109 (90), 80 (57), 52 (100). The filtrate was evaporated to give a residue $(184 \mathrm{mg})$ which consisted of three compounds, $N$ - $t$ butyl- $N^{\prime}$-(5-tropolonyl)urea (3), $N, N^{\prime}$-di(t-butyl)urea, and $\mathbf{1}$ in a ratio of $c a$. $1: 0.7: 0.5$ estimated by the ${ }^{1} \mathrm{H}-\mathrm{NMR}$ integration of the respective $\mathrm{NH}$ groups.

Reaction of $\mathbf{1}$ with $\mathbf{3}$ eq of $\boldsymbol{t}$-Butyl Isocyanate A solution of $\mathbf{1}(411 \mathrm{mg}$, $3.00 \mathrm{mmol})$ and $t$-butyl isocyanate $(1.06 \mathrm{ml}, 9.00 \mathrm{mmol})$ in THF $(10 \mathrm{ml})$ was refluxed for $3 \mathrm{~h}$, then cooled to room temperature and the solvent was evaporated in vacuo. The residue was flash-chromatographed [silica gel, $\mathrm{MeOH}-\mathrm{CH}_{2} \mathrm{Cl}_{2}(1: 9)$ ] to give $3(466 \mathrm{mg}, 66 \%)$ as brown scales $(\mathrm{MeOH}-$ hexane), mp $207-210^{\circ} \mathrm{C} .{ }^{1} \mathrm{H}-\mathrm{NMR}$ (DMSO-d $)_{6} \delta: 8.50(1 \mathrm{H}$, brs, NH), $7.51(2 \mathrm{H}, \mathrm{dd}, J=11.0,1.5 \mathrm{~Hz}), 7.17(2 \mathrm{H}, \mathrm{dd}, J=11.0,1.5 \mathrm{~Hz}), 6.13(1 \mathrm{H}, \mathrm{br}$, $\mathrm{NH}), 1.27\left(9 \mathrm{H}, \mathrm{s}, t\right.$-Bu). ${ }^{13} \mathrm{C}-\mathrm{NMR}$ (DMSO- $\left.d_{6}\right) \delta: 168.0,153.8,140.9$, 126.3, 125.2, 49.7, 28.9. IR (KBr) cm $\mathrm{cm}^{-1}: 3350,3170,3005,1640,1560$, 1446, 1359, 1260. HR-MS $m / z$ : 236.1163 (Calcd for $\mathrm{C}_{12} \mathrm{H}_{16} \mathrm{~N}_{2} \mathrm{O}_{3}$ : 236.1160). MS $m / z$ : $236\left(\mathrm{M}^{+}, 9\right), 163$ (13), 137 (100).

Reaction of $\boldsymbol{N}$ - $t$-Butyl- $\boldsymbol{N}^{\prime}$-(5-tropolonyl)urea (3) with 1 A solution of $3(253 \mathrm{mg}, 1.07 \mathrm{mmol})$ and $\mathbf{1}(293 \mathrm{mg}, 2.14 \mathrm{mmol})$ in toluene $(10 \mathrm{ml})$ was heated at $120^{\circ} \mathrm{C}$ for $14 \mathrm{~h}$, then cooled to room temperature and the solvent was evaporated in vacuo. The residue was washed with $\mathrm{MeOH}$ to give 2 (264 mg, 82\%).

Preparation of $\boldsymbol{N}$-(1-Adamantyl)- $\boldsymbol{N}^{\prime}$-(5-tropolonyl)urea A solution of $1(206 \mathrm{mg}, 1.50 \mathrm{mmol})$ and 1 -adamantyl isocyanate $(531 \mathrm{mg}, 3.00 \mathrm{mmol})$ in THF $(10 \mathrm{ml})$ was refluxed for $28 \mathrm{~h}$, then cooled to room temperature and the solvent was evaporated in vacuo. The residue was flash-chromatographed [silica gel, $\mathrm{CH}_{2} \mathrm{Cl}_{2}-1,2$-dimethoxyethane $\left.(20: 1)\right]$ to give $\mathrm{N}$-(1adamantyl)- $N^{\prime}$-(5-tropolonyl)urea $(168 \mathrm{mg}, 36 \%)$ as brown scales $(\mathrm{MeOH})$, $\mathrm{mp} 214-216^{\circ} \mathrm{C}$ and $230-235^{\circ} \mathrm{C}$ (dec.). ${ }^{1} \mathrm{H}-\mathrm{NMR}$ (DMSO- $d_{6}$ ) $\delta: 8.51$ $(1 \mathrm{H}, \mathrm{brs}, \mathrm{NH}), 7.50(2 \mathrm{H}, \mathrm{d}, J=12.3 \mathrm{~Hz}), 7.17(2 \mathrm{H}, \mathrm{d}, J=12.3 \mathrm{~Hz}), 6.01(1 \mathrm{H}$, brs, NH), $2.06-1.98(3 \mathrm{H}, \mathrm{m}), 1.94-1.89(6 \mathrm{H}, \mathrm{m}), 1.64-1.59(6 \mathrm{H}, \mathrm{m}) . \mathrm{IR}$ $(\mathrm{KBr}) \mathrm{cm}^{-1}: 3370,2905,1664,1529,1447$. HR-MS m/z: 314.1617 (Calcd for $\left.\mathrm{C}_{18} \mathrm{H}_{22} \mathrm{~N}_{2} \mathrm{O}_{3}: 314.1629\right)$. MS $m / z: 314\left(\mathrm{M}^{+}, 0.3\right), 163$ (20), 151 (17), 135 (17), 107 (15), 94 (100).

Tropolonylureas 4a-s; General Procedure A solution of 3 (0.5 $\mathrm{mmol})$ and amine $(1-3 \mathrm{eq})$ in toluene $(5 \mathrm{ml})$ was heated at $120^{\circ} \mathrm{C}$. The reaction was monitored by NMR (or TLC). When the reaction reached completion, the solvent was evaporated in vacuo. The residue was washed with $\mathrm{MeOH}$ (or $\mathrm{CH}_{2} \mathrm{Cl}_{2}$, ether, or $\mathrm{H}_{2} \mathrm{O}$ ), and the crude product was collected by filtration. If necessary, the residue was purified by flash chromatography (silica gel or aluminum oxide).

$\boldsymbol{N}$-(2,6-Dimethylphenyl)- $\boldsymbol{N}^{\prime}$-(5-tropolonyl)urea (4a) Dark brown scales $(\mathrm{MeOH}), \mathrm{mp} 258-260{ }^{\circ} \mathrm{C} .{ }^{1} \mathrm{H}-\mathrm{NMR}\left(\mathrm{DMSO}-d_{6}\right) \delta: 9.01(1 \mathrm{H}, \mathrm{brs}$, $\mathrm{NH}), 7.87(1 \mathrm{H}$, br s, NH), $7.59(2 \mathrm{H}, \mathrm{d}, J=12.3 \mathrm{~Hz}), 7.20(2 \mathrm{H}, \mathrm{d}, J=12.3 \mathrm{~Hz})$, $7.01(3 \mathrm{H}, \mathrm{s}), 2.19(6 \mathrm{H}, \mathrm{s}, \mathrm{Me} \times 2)$. HR-MS $m / z: 284.1163$ (Calcd for $\left.\mathrm{C}_{16} \mathrm{H}_{16} \mathrm{~N}_{2} \mathrm{O}_{3}: 284.1160\right)$. MS $m / z: 284\left(\mathrm{M}^{+}, 4\right), 163$ (53), 135 (49), 121 (100).

$\boldsymbol{N}$-(4-Methylphenyl)- $\boldsymbol{N}^{\prime}$-(5-tropolonyl)urea (4b) Pale yellow needles $(\mathrm{MeOH}), \operatorname{mp} 260-262{ }^{\circ} \mathrm{C}$ (dec.). ${ }^{1} \mathrm{H}-\mathrm{NMR}$ (DMSO- $\left.d_{6}\right) \delta: 8.85$ (1H, br s, $\mathrm{NH}), 8.68(1 \mathrm{H}$, br s, NH), $7.56(2 \mathrm{H}, \mathrm{d}, J=12.3 \mathrm{~Hz}), 7.32(2 \mathrm{H}, \mathrm{d}, J=8.7 \mathrm{~Hz})$, $7.20(2 \mathrm{H}, \mathrm{d}, J=12.3 \mathrm{~Hz}), 7.08(2 \mathrm{H}, \mathrm{d}, J=8.7 \mathrm{~Hz}), 2.23(3 \mathrm{H}, \mathrm{s}, \mathrm{Me})$. IR (KBr) $\mathrm{cm}^{-1}: 3300,3210,1683,1605,1544,1449,1425,1355$. HR-MS $m / z$ : 270.0997 (Calcd for $\mathrm{C}_{15} \mathrm{H}_{14} \mathrm{~N}_{2} \mathrm{O}_{3}:$ 270.1004). MS m/z: $270\left(\mathrm{M}^{+}, 21\right), 163$ (19), 137 (77), 109 (100).

$\boldsymbol{N}$-(3,4-Difluorophenyl)- $\boldsymbol{N}^{\prime}$-(5-tropolonyl)urea (4c) Ocher needles $(\mathrm{MeOH}), \operatorname{mp} 259-261{ }^{\circ} \mathrm{C}$ (dec.). ${ }^{1} \mathrm{H}-\mathrm{NMR}$ (DMSO- $\left.d_{6}\right) \delta: 9.01(1 \mathrm{H}$, br s, $\mathrm{NH}), 8.95(1 \mathrm{H}$, br s, NH), $7.64(1 \mathrm{H}, \mathrm{ddd}, J=13.3,7.5,2.4 \mathrm{~Hz}), 7.54(2 \mathrm{H}, \mathrm{d}$, $J=12.0 \mathrm{~Hz}), 7.34(1 \mathrm{H}$, ddd, $J=10.0,9.0,9.0 \mathrm{~Hz}), 7.20(2 \mathrm{H}, \mathrm{d}, J=12.0 \mathrm{~Hz})$, $7.15-7.10(1 \mathrm{H}, \mathrm{m})$. HR-MS $m / z$ : 292.0646 (Calcd for $\mathrm{C}_{14} \mathrm{H}_{10} \mathrm{~F}_{2} \mathrm{~N}_{2} \mathrm{O}_{3}$ :
292.0659). MS m/z: $292\left(\mathrm{M}^{+}, 10\right), 163$ (52), 155 (54), 137 (40), 135 (45), 129 (100), 127 (39).

$\boldsymbol{N}$-Methyl- $\boldsymbol{N}$-phenyl- $\boldsymbol{N}^{\prime}$-(5-tropolonyl)urea (4d) Ocher prisms (AcOEt), mp $134-136{ }^{\circ} \mathrm{C} .{ }^{1} \mathrm{H}-\mathrm{NMR}$ (DMSO-d $\left.{ }_{6}\right) \delta: 8.37(1 \mathrm{H}$, br s, NH), $7.46(2 \mathrm{H}, \mathrm{dd}, J=11.1,1.5 \mathrm{~Hz}), 7.41(2 \mathrm{H}, \mathrm{dd}, J=7.2,7.2 \mathrm{~Hz}), 7.33(2 \mathrm{H}$, dd, $J=7.2,1.2 \mathrm{~Hz}), 7.25(1 \mathrm{H}, \mathrm{tt}, J=7.2,1.2 \mathrm{~Hz}), 7.14(2 \mathrm{H}, \mathrm{dd}, J=11.1,1.5 \mathrm{~Hz})$, $3.25(3 \mathrm{H}, \mathrm{s}, \mathrm{OMe}) .{ }^{13} \mathrm{C}-\mathrm{NMR}$ (DMSO- $\left.d_{6}\right) \delta: 168.8,154.7,143.5,139.9$, 130.1, 129.1, 126.2, 125.9, 124.0, 39.7. IR (KBr) $\mathrm{cm}^{-1}:$ 3400, 3225, 1684, $1543,1496,1445,1363,1262$. HR-MS $m / z$ : 270.1015 (Calcd for $\left.\mathrm{C}_{15} \mathrm{H}_{14} \mathrm{~N}_{2} \mathrm{O}_{3}: 270.1004\right)$. MS $m / z: 270\left(\mathrm{M}^{+}, 27\right), 163$ (29), 134 (82), 106 (100).

$\boldsymbol{N}$-(8-Quinolyl)- $\boldsymbol{N}^{\prime}$-(5-tropolonyl)urea (4e) Yellow needles $(\mathrm{MeOH})$, mp $246-248^{\circ} \mathrm{C} .{ }^{1} \mathrm{H}-\mathrm{NMR}\left(\mathrm{DMSO}-d_{6}\right) \delta$ : $10.1(1 \mathrm{H}, \mathrm{brs}, \mathrm{NH}), 9.78(1 \mathrm{H}$, br s, NH), $8.93(1 \mathrm{H}, \mathrm{dd}, J=4.4,1.8 \mathrm{~Hz}), 8.53(1 \mathrm{H}, \mathrm{dd}, J=6.0,3.0 \mathrm{~Hz}), 8.40$ $(1 \mathrm{H}, \mathrm{dd}, J=8.6,1.8 \mathrm{~Hz}), 7.69(2 \mathrm{H}, \mathrm{dd}, J=11.0,1.2 \mathrm{~Hz}), 7.64(1 \mathrm{H}, \mathrm{dd}, J=8.6$, $4.4 \mathrm{~Hz}), 7.59-7.52(2 \mathrm{H}, \mathrm{m}), 7.26(2 \mathrm{H}, \mathrm{dd}, J=11.0,1.2 \mathrm{~Hz}) .{ }^{13} \mathrm{C}-\mathrm{NMR}$ $\left(\mathrm{DMSO}_{6}\right) \delta: 168.6,152.1,148.2,139.6,137.5,136.5,135.3,127.7,127.2$, $127.0,125.0,122.0,120.0,114.4$. IR (KBr) cm $\mathrm{cm}^{-1}: 3315,3215,3080,1712$, $1571,1548,1525,1458,1356$. HR-MS $m / z$ : 307.0962 (Calcd for $\mathrm{C}_{17} \mathrm{H}_{13} \mathrm{~N}_{3} \mathrm{O}_{3}$ : 307.0956). MS m/z: $307\left(\mathrm{M}^{+}, 1\right), 170$ (53), 163 (27), 144 (100).

$\boldsymbol{N}$-(5-Indolyl)- $\boldsymbol{N}^{\prime}$-(5-tropolonyl)urea (4f) Ocher needles $(\mathrm{MeOH}), \mathrm{mp}$ $238-240{ }^{\circ} \mathrm{C} .{ }^{1} \mathrm{H}-\mathrm{NMR}\left(\mathrm{DMSO}-d_{6}\right) \delta: 10.9(1 \mathrm{H}$, br s, indole $\mathrm{NH}), 8.82(1 \mathrm{H}$, brs, NH), $8.54(1 \mathrm{H}$, brs, NH), $7.66(1 \mathrm{H}, \mathrm{d}, J=2.1 \mathrm{~Hz}), 7.59(2 \mathrm{H}, \mathrm{d}$, $J=12.0 \mathrm{~Hz}), 7.32-7.26(2 \mathrm{H}, \mathrm{m}), 7.21(2 \mathrm{H}, \mathrm{d}, J=12.0 \mathrm{~Hz}), 7.07(1 \mathrm{H}, \mathrm{dd}$, $J=8.9,2.1 \mathrm{~Hz}), 6.38-6.32(1 \mathrm{H}, \mathrm{m}) . \mathrm{IR}(\mathrm{KBr}) \mathrm{cm}^{-1}: 3265,1628,1613$, $1562,1535,1448,1353$. HR-MS $m / z$ : 295.0942 (Calcd for $\mathrm{C}_{16} \mathrm{H}_{13} \mathrm{~N}_{3} \mathrm{O}_{3}$ : 295.0956). MS m/z: $295\left(\mathrm{M}^{+}, 20\right), 164$ (20), 163 (82), 137 (100).

$\boldsymbol{N}$-(3-Coumarinyl)- $\boldsymbol{N}^{\prime}$-(5-tropolonyl)urea (4g) Ocher powder $(\mathrm{MeOH})$, mp $288-290{ }^{\circ} \mathrm{C}$ (dec.). ${ }^{1} \mathrm{H}-\mathrm{NMR}\left(\mathrm{DMSO}-d_{6}\right) \delta: 9.66(1 \mathrm{H}$, br s, NH), 8.89 $(1 \mathrm{H}$, brs, NH), $8.43(1 \mathrm{H}, \mathrm{s}), 7.67(1 \mathrm{H}, \mathrm{d}, J=7.8 \mathrm{~Hz}), 7.56(2 \mathrm{H}, \mathrm{d}$, $J=12.0 \mathrm{~Hz}), 7.47(1 \mathrm{H}, \mathrm{dd}, J=7.8,7.8 \mathrm{~Hz}), 7.39(1 \mathrm{H}, \mathrm{d}, J=7.8 \mathrm{~Hz}), 7.32(1 \mathrm{H}$, dd, $J=7.8,7.8 \mathrm{~Hz}), 7.20(2 \mathrm{H}, \mathrm{d}, J=12.0 \mathrm{~Hz}) . \mathrm{IR}(\mathrm{KBr}) \mathrm{cm}^{-1}: 3340,1674$, 1555, 1449. HR-MS $m / z: 324.0731$ (Calcd for $\mathrm{C}_{17} \mathrm{H}_{12} \mathrm{~N}_{2} \mathrm{O}_{5}: 324.0745$ ). MS $m / z: 324\left(\mathrm{M}^{+}, 5\right), 187(76), 161(100)$.

$\boldsymbol{N}$-(2-Benzothiazolyl)- $\boldsymbol{N}^{\prime}$-(5-tropolonyl)urea (4h) Pale yellow powder $(\mathrm{MeOH}), \mathrm{mp} 257-260{ }^{\circ} \mathrm{C}$ (dec.). ${ }^{1} \mathrm{H}-\mathrm{NMR}$ (DMSO-d $)_{6} \delta: 11.4-10.0(1 \mathrm{H}$, br s, NH), $9.37(1 \mathrm{H}$, br s, NH), $7.89(1 \mathrm{H}$, brd, $J=7.8 \mathrm{~Hz}), c a .7 .65-7.58$ $(1 \mathrm{H}, \mathrm{m}), 7.64(2 \mathrm{H}, \mathrm{d}, J=12.3 \mathrm{~Hz}), 7.39(1 \mathrm{H}, \mathrm{ddd}, J=7.8,7.8,1.2 \mathrm{~Hz})$, $7.27-7.20(1 \mathrm{H}, \mathrm{m}), 7.24(2 \mathrm{H}, \mathrm{d}, J=12.3 \mathrm{~Hz}) . \mathrm{IR}(\mathrm{KBr}) \mathrm{cm}^{-1}: 3350,3265$, 2990, 1710, 1552, 1444, 1350. HR-MS m/z: 313.0509 (Calcd for $\left.\mathrm{C}_{15} \mathrm{H}_{11} \mathrm{~N}_{3} \mathrm{O}_{3} \mathrm{~S}: 313.0520\right)$. MS m/z: $313\left(\mathrm{M}^{+}, 0.6\right), 176$ (100), 163 (33), 150 (91).

$\boldsymbol{N}$-(5-Bromo-2-pyridyl)- $\boldsymbol{N}^{\prime}$-(5-tropolonyl)urea (4i) Dark brown powder $(\mathrm{MeOH}), \operatorname{mp} 240-242{ }^{\circ} \mathrm{C} .{ }^{1} \mathrm{H}-\mathrm{NMR}$ (DMSO- $\left.d_{6}\right) \delta: 10.0(1 \mathrm{H}$, br s, NH), $9.61(1 \mathrm{H}$, br s, NH), $8.39(1 \mathrm{H}, \mathrm{d}, J=2.4 \mathrm{~Hz}), 7.49(1 \mathrm{H}, \mathrm{dd}, J=8.7,2.4 \mathrm{~Hz})$, $7.61(2 \mathrm{H}, \mathrm{d}, J=12.3 \mathrm{~Hz}), 7.61(1 \mathrm{H}, \mathrm{d}, J=8.7 \mathrm{~Hz}), 7.22(2 \mathrm{H}, \mathrm{d}, J=12.3 \mathrm{~Hz})$. HR-MS $m / z$ : 336.9872, 334.9919 (Calcd for $\mathrm{C}_{13} \mathrm{H}_{10} \mathrm{BrN}_{3} \mathrm{O}_{3}$ : 336.9886, 334.9905). MS m/z: 337, $335\left(\mathrm{M}^{+}, 19,20\right), 200$ (51), 198 (51), 174 (96), $172(100)$.

$\boldsymbol{N}$-(2-Chloro-5-pyridyl)- $\boldsymbol{N}^{\prime}$-(5-tropolonyl)urea (4j) Ocher needles $(\mathrm{MeOH}), \mathrm{mp} 248-250{ }^{\circ} \mathrm{C}$ (dec.). ${ }^{1} \mathrm{H}-\mathrm{NMR}$ (DMSO-d $) \delta: 9.41(1 \mathrm{H}$, brs, $\mathrm{NH}), 9.29(1 \mathrm{H}$, br s, NH), $8.50(1 \mathrm{H}, \mathrm{d}, J=2.7 \mathrm{~Hz}), 7.98(1 \mathrm{H}, \mathrm{dd}, J=8.7$, $2.7 \mathrm{~Hz}), 7.54(2 \mathrm{H}, \mathrm{d}, J=12.3 \mathrm{~Hz}), 7.42(1 \mathrm{H}, \mathrm{d}, J=8.7 \mathrm{~Hz}), 7.17(2 \mathrm{H}, \mathrm{d}$, $J=12.3 \mathrm{~Hz}$ ). HR-MS $m / z: 293.0370,291.0404$ (Calcd for $\mathrm{C}_{13} \mathrm{H}_{10} \mathrm{ClN}_{3} \mathrm{O}_{3}$ : 293.0380, 291.0410). MS m/z: 293, $291\left(\mathrm{M}^{+}, 1,4\right), 163$ (49), 154 (100).

$\boldsymbol{N}$-Pyrazinyl- $\boldsymbol{N}^{\prime}$-(5-tropolonyl)urea (4k) Brown powder $(\mathrm{MeOH}), \mathrm{mp}$ $258-260{ }^{\circ} \mathrm{C} .{ }^{1} \mathrm{H}-\mathrm{NMR}\left(\mathrm{DMSO}-d_{6}\right) \delta: 9.77(1 \mathrm{H}$, br s, NH), $9.68(1 \mathrm{H}$, br s, $\mathrm{NH}), 9.00(1 \mathrm{H}, \mathrm{d}, J=1.2 \mathrm{~Hz}), 8.32(1 \mathrm{H}, \mathrm{dd}, J=2.7,1.2 \mathrm{~Hz}), 8.27(1 \mathrm{H}, \mathrm{d}$, $J=2.7 \mathrm{~Hz}), 7.61(2 \mathrm{H}, \mathrm{d}, J=12.0 \mathrm{~Hz}), 7.23(2 \mathrm{H}, \mathrm{d}, J=12.0 \mathrm{~Hz}) . \mathrm{IR}(\mathrm{KBr})$ $\mathrm{cm}^{-1}: 3025,1695,1604,1549,1503,1451,1419,1399,1362$. HR-MS $m / z$ : 258.0752 (Calcd for $\left.\mathrm{C}_{12} \mathrm{H}_{10} \mathrm{~N}_{4} \mathrm{O}_{3}: 258.0752\right)$. MS $m / z: 258\left(\mathrm{M}^{+}, 28\right), 163$ (41), 137 (55), 121 (58), 109 (100), 95 (86).

1-(5-Tropolonylcarbamoyl)piperazine (4l) Pale yellow powder $\left(\mathrm{CH}_{2} \mathrm{Cl}_{2}-\mathrm{MeOH}\right), \mathrm{mp} 232-235^{\circ} \mathrm{C}(\mathrm{dec}) .{ }^{1} \mathrm{H}-\mathrm{NMR}$ (DMSO- $\left.d_{6}\right) \quad \delta: 8.61$ $(1 \mathrm{H}$, br s, NH), $7.44(2 \mathrm{H}, \mathrm{d}, J=12.0 \mathrm{~Hz}), 7.14(2 \mathrm{H}, \mathrm{d}, J=12.0 \mathrm{~Hz}), 3.34(4 \mathrm{H}$, $\mathrm{t}, J=5.1 \mathrm{~Hz}), 2.67(4 \mathrm{H}, \mathrm{t}, J=5.1 \mathrm{~Hz})$. HR-MS $m / z$ : 249.1124 (Calcd for $\left.\mathrm{C}_{12} \mathrm{H}_{15} \mathrm{~N}_{3} \mathrm{O}_{3}: 249.1112\right)$. MS m/z: $249\left(\mathrm{M}^{+}, 1\right), 163$ (100), 135 (71), 107 (50), 86 (39), 79 (34), 52 (58), 44 (94).

4-(2-Hydoxyethyl)-1-(5-tropolonylcarbamoyl)piperazine (4m) Yellow prisms, mp $93-95^{\circ} \mathrm{C}$, then solidify and melt at $200-202{ }^{\circ} \mathrm{C}$. ${ }^{1} \mathrm{H}-\mathrm{NMR}$ $\left(\mathrm{CDCl}_{3}\right) \delta: 7.43(2 \mathrm{H}, \mathrm{dd}, J=10.8,1.8 \mathrm{~Hz}), 7.30(2 \mathrm{H}, \mathrm{dd}, J=10.8,1.8 \mathrm{~Hz})$, $6.40(1 \mathrm{H}, \mathrm{br} \mathrm{s}), 3.68(2 \mathrm{H}, \mathrm{t}, J=5.1 \mathrm{~Hz}), 3.54(4 \mathrm{H}, \mathrm{t}, J=5.1 \mathrm{~Hz}), 2.62(2 \mathrm{H}, \mathrm{t}$, $J=5.1 \mathrm{~Hz}), 2.59(4 \mathrm{H}, \mathrm{t}, J=5.1 \mathrm{~Hz})$. HR-MS $m / z: 293.1344$ (Calcd for 
$\mathrm{C}_{14} \mathrm{H}_{19} \mathrm{~N}_{3} \mathrm{O}_{4}:$ 293.1374). MS m/z: $293\left(\mathrm{M}^{+}, 4\right), 163$ (59), 135 (55), 99 (100). 4-Methyl-1-(5-tropolonylcarbamoyl)homopiperazine (4n) Yellow powder, mp 105-107 and $175-177^{\circ} \mathrm{C} .{ }^{1} \mathrm{H}-\mathrm{NMR}\left(\mathrm{CDCl}_{3}\right) \delta: 7.46(2 \mathrm{H}, \mathrm{d}$, $J=12.3 \mathrm{~Hz}), 7.30(2 \mathrm{H}, \mathrm{d}, J=12.3 \mathrm{~Hz}), 6.33(1 \mathrm{H}, \mathrm{br} \mathrm{s}, \mathrm{NH}), 3.72-3.62(2 \mathrm{H}$, $\mathrm{m}), 3.60(2 \mathrm{H}, \mathrm{t}, J=6.0 \mathrm{~Hz}), 2.74-2.66(2 \mathrm{H}, \mathrm{m}), 2.66-2.58(2 \mathrm{H}, \mathrm{m}), 2.41$ $(3 \mathrm{H}, \mathrm{s}, \mathrm{Me}), 2.00(2 \mathrm{H}, \mathrm{m})$. HR-MS m/z: 277.1430 (Calcd for $\mathrm{C}_{14} \mathrm{H}_{19} \mathrm{~N}_{3} \mathrm{O}_{3}$ : 277.1425). MS m/z: 277 (M+ , 5), 163 (54), 135 (47), 114 (22), 107 (38), 58 (100)

4-(5-Tropolonylcarbamoyl)morpholine (40) Pale yellow powder $(\mathrm{MeOH}), \mathrm{mp} 213-215^{\circ} \mathrm{C} .{ }^{1} \mathrm{H}-\mathrm{NMR}\left(\mathrm{CDCl}_{3}\right) \delta: 7.43(2 \mathrm{H}, \mathrm{d}, J=12.3 \mathrm{~Hz})$, $7.29(2 \mathrm{H}, \mathrm{d}, J=12.3 \mathrm{~Hz}), 6.43(1 \mathrm{H}$, br s, NH), $3.76(4 \mathrm{H}, \mathrm{t}, J=4.5 \mathrm{~Hz}), 3.50$ $\left(4 \mathrm{H}, \mathrm{t}, J=4.5 \mathrm{~Hz}\right.$ ) HR-MS $m / z: 250.0951$ (Calcd for $\mathrm{C}_{12} \mathrm{H}_{14} \mathrm{~N}_{2} \mathrm{O}_{4}: 250.0953$ ). MS m/z: 250 (M $\left.\mathrm{M}^{+}, 26\right), 163$ (56), 135 (58), 114 (81), 107 (45), 70 (100).

$\boldsymbol{N}$-[2-(Dimethylamino)ethyl]- $\boldsymbol{N}$-methyl- $\boldsymbol{N}^{\prime}$-(5-tropolonyl)urea (4p) Ocher powder, mp $108-110{ }^{\circ} \mathrm{C} .{ }^{1} \mathrm{H}-\mathrm{NMR}\left(\mathrm{CDCl}_{3}\right) \delta: 11.0(1 \mathrm{H}$, br s, NH), $7.39(2 \mathrm{H}, \mathrm{d}, J=12.6 \mathrm{~Hz}), 7.30(2 \mathrm{H}, \mathrm{d}, J=12.6 \mathrm{~Hz}), 3.38(2 \mathrm{H}, \mathrm{t}, J=4.2 \mathrm{~Hz})$, $2.99(3 \mathrm{H}, \mathrm{s}, \mathrm{Me}), 2.63(2 \mathrm{H}, \mathrm{t}, J=4.2 \mathrm{~Hz}), 2.42(6 \mathrm{H}, \mathrm{s}, \mathrm{Me} \times 2)$. HR-MS $m / z$ 265.1414 (Calcd for $\left.\mathrm{C}_{13} \mathrm{H}_{19} \mathrm{~N}_{3} \mathrm{O}_{3}: 265.1425\right)$. MS m/z: $265\left(\mathrm{M}^{+}, 1.4\right), 163$ (15), 135 (16), 107 (13), 79 (19), 58 (100).

$\boldsymbol{N}$-[3-(4-Morpholinopropyl)]- $\boldsymbol{N}^{\prime}$-(5-tropolonyl)urea (4q) Ocher powder, mp $186-188^{\circ} \mathrm{C} .{ }^{1} \mathrm{H}-\mathrm{NMR}\left(\mathrm{CD}_{3} \mathrm{OD}\right) \delta: 7.62(2 \mathrm{H}, \mathrm{dd}, J=11.1,1.5 \mathrm{~Hz})$, $7.30(2 \mathrm{H}, \mathrm{dd}, J=11.1,1.5 \mathrm{~Hz}), 3.69(4 \mathrm{H}, \mathrm{t}, J=4.5 \mathrm{~Hz}), 3.24(2 \mathrm{H}, \mathrm{t}$, $J=6.9 \mathrm{~Hz}), 2.48(4 \mathrm{H}, \mathrm{t}, J=4.5 \mathrm{~Hz}), 2.43(2 \mathrm{H}, \mathrm{t}, J=7.5 \mathrm{~Hz}), 1.74(2 \mathrm{H}, \mathrm{tt}$, $J=7.5,6.9 \mathrm{~Hz}$ ). HR-MS $m / z$ : 307.1544 (Calcd for $\mathrm{C}_{15} \mathrm{H}_{21} \mathrm{~N}_{3} \mathrm{O}_{4}: 307.1531$ ). MS m/z: $307\left(\mathrm{M}^{+}, 0.2\right), 171$ (15), 163 (41), 135 (39), 100 (100).

$\boldsymbol{N}$-[2-(4-1H-Imidazolyl)ethyl]- $\boldsymbol{N}^{\prime}$-(5-tropolonyl)urea (4r) Yellow powder (AcOEt), mp $190-192{ }^{\circ} \mathrm{C} .{ }^{1} \mathrm{H}-\mathrm{NMR} \quad\left(\mathrm{CD}_{3} \mathrm{OD}\right) \quad \delta: 7.60 \quad(1 \mathrm{H}, \mathrm{d}$, $J=1.2 \mathrm{~Hz}), 7.57(2 \mathrm{H}, \mathrm{dd}, J=10.8,1.5 \mathrm{~Hz}), 7.27(2 \mathrm{H}, \mathrm{dd}, J=10.8,1.5 \mathrm{~Hz})$, $6.87(1 \mathrm{H}, \mathrm{d}, J=1.2 \mathrm{~Hz}), 3.45(2 \mathrm{H}, \mathrm{t}, J=6.6 \mathrm{~Hz}), 2.80(2 \mathrm{H}, \mathrm{t}, J=6.6 \mathrm{~Hz}) . \mathrm{IR}$ $(\mathrm{KBr}) \mathrm{cm}^{-1}: 3210,1664,1504,1432,1344$. MS m/z: $163\left(\mathrm{M}^{+},-111,52\right)$, 137 (15), 135 (50), 107 (43), 82 (100).

$N$-[2-Hydroxy-3-(4-1H-indolyloxy)propyl]- $N$-(2-propyl)- $N^{\prime}-(5-$ tropolonyl)urea (4s) Light brown prisms (MeOH-AcOEt-hexane), mp $163-165^{\circ} \mathrm{C} .{ }^{1} \mathrm{H}-\mathrm{NMR}\left(\mathrm{CD}_{3} \mathrm{OD}\right) \delta: 7.46(2 \mathrm{H}, \mathrm{dd}, J=10.4,1.5 \mathrm{~Hz}), 7.27$ $(2 \mathrm{H}, \mathrm{dd}, J=10.4,1.5 \mathrm{~Hz}), 7.11(1 \mathrm{H}, \mathrm{d}, J=3.3 \mathrm{~Hz}), 7.40-6.97(2 \mathrm{H}, \mathrm{m})$, $6.55-6.49(1 \mathrm{H}, \mathrm{m}), 6.51(1 \mathrm{H}, \mathrm{d}, J=3.3 \mathrm{~Hz}), 4.46(1 \mathrm{H}, \mathrm{qq}, J=6.9,6.9 \mathrm{~Hz})$, $4.30-4.14(1 \mathrm{H}, \mathrm{m}), 4.20(1 \mathrm{H}, \mathrm{dd}, J=9.3,4.8 \mathrm{~Hz}), 4.09(1 \mathrm{H}, \mathrm{dd}, J=9.3$, $6.9 \mathrm{~Hz}), 3.67(1 \mathrm{H}, \mathrm{dd}, J=16.1,1.8 \mathrm{~Hz}), 3.51(1 \mathrm{H}, \mathrm{dd}, J=16.1,8.4 \mathrm{~Hz}), 1.26$ $(3 \mathrm{H}, \mathrm{d}, J=6.9 \mathrm{~Hz}), 1.21(3 \mathrm{H}, \mathrm{d}, J=6.9 \mathrm{~Hz}) . \mathrm{IR}(\mathrm{KBr}) \mathrm{cm}^{-1}: 3230,1672$, 1564, 1500, 1436, 1352, 1209, 1080. MS m/z: $248\left(\mathrm{M}^{+},-163,9\right), 204$ (5), 163 (16), 133 (89), 104 (24), 72 (100).

Phenylureas $(\mathbf{6 a}-\mathbf{c})$ and 3 -pyridylureas $(\mathbf{8 a}-\mathbf{b})$ were obtained by a procedure similar to that described for tropolonylureas (4).

Preparation of $\boldsymbol{N}$ - $t$-Butyl- $\boldsymbol{N}^{\prime}$-phenylurea (5) A solution of $t$-butylamine $(1.06 \mathrm{ml}, 10.0 \mathrm{mmol})$ and phenyl isocyanate $(1.08 \mathrm{ml}, 10.0 \mathrm{mmol})$ in benzene $(10 \mathrm{ml})$ was stirred for $2 \mathrm{~h}$ at room temperature, and the resulting precipitate was collected by filtration to afford $5(1.14 \mathrm{~g}, 59 \%)$ as colorless prisms (benzene), mp $167-169^{\circ} \mathrm{C}$ (lit., ${ }^{35)} 165^{\circ} \mathrm{C}$ ). ${ }^{1} \mathrm{H}-\mathrm{NMR}$ (DMSO- $d_{6}$ ) $\delta$ : $8.18(1 \mathrm{H}$, br s, NH), $7.32(2 \mathrm{H}, \mathrm{d}, J=7.5 \mathrm{~Hz}), 7.18(2 \mathrm{H}, \mathrm{dd}, J=7.5,7.5 \mathrm{~Hz})$, $6.85(1 \mathrm{H}, \mathrm{t}, J=7.5 \mathrm{~Hz}), 5.94(1 \mathrm{H}, \mathrm{brs}, \mathrm{NH}), 1.27(9 \mathrm{H}, \mathrm{s}, t-\mathrm{Bu})$. MS $m / z: 192$ $\left(\mathrm{M}^{+}, 42\right), 135(16), 94(75)$.

$\boldsymbol{N}$-Phenyl- $\boldsymbol{N}^{\prime}$-(5-tropolonyl)urea (6a) Brown scales $(\mathrm{MeOH}), \mathrm{mp}$ $255-258{ }^{\circ} \mathrm{C}$ (dec.). ${ }^{1} \mathrm{H}-\mathrm{NMR}$ (DMSO- $\left.d_{6}\right) \delta: 8.89(1 \mathrm{H}, \mathrm{brs}, \mathrm{NH}), 8.79(1 \mathrm{H}$, br s, NH), $7.56(2 \mathrm{H}, \mathrm{d}, J=12.3 \mathrm{~Hz}), 7.44(2 \mathrm{H}, \mathrm{dd}, J=7.8,0.6 \mathrm{~Hz}), 7.28(2 \mathrm{H}$, dd, $J=7.8,7.8 \mathrm{~Hz}), 7.21(2 \mathrm{H}, \mathrm{d}, J=12.3 \mathrm{~Hz}), 6.98(1 \mathrm{H}, \mathrm{tt}, J=7.8,0.6 \mathrm{~Hz})$. HR-MS $m / z$ : 256.0847 (Calcd for $\mathrm{C}_{14} \mathrm{H}_{12} \mathrm{~N}_{2} \mathrm{O}_{3}: 256.0847$ ). MS $m / z: 256$ $\left(\mathrm{M}^{+}, 14\right), 163$ (96), 137 (70), 135 (86), 109 (100).

1-(Phenylcarbamoyl)piperazine (6b) In order to avoid the formation of 1,4-bis(phenylcarbamoyl)piperazine (reaction of piperazine with one or two ${ }^{36)}$ equivalents of phenyl isocyanate gave this compound), an excess amount of piperazine (15 eq) was used to afford $\mathbf{6 b}$ as white granules, mp $114-115^{\circ} \mathrm{C} .{ }^{1} \mathrm{H}-\mathrm{NMR}\left(\mathrm{CDCl}_{3}\right) \delta: 7.35(2 \mathrm{H}, \mathrm{dd}, J=7.2,1.2 \mathrm{~Hz}), 7.31(2 \mathrm{H}$, dd, $J=7.2,7.2 \mathrm{~Hz}), 7.04(1 \mathrm{H}, \mathrm{tt}, J=7.2,1.2 \mathrm{~Hz}), 6.30(1 \mathrm{H}$, br s, NH), 3.47 $(4 \mathrm{H}, \mathrm{t}, J=5.1 \mathrm{~Hz}), 2.92(4 \mathrm{H}, \mathrm{t}, J=5.1 \mathrm{~Hz})$. HR-MS $m / z: 205.1206$ (Calcd for $\left.\mathrm{C}_{11} \mathrm{H}_{15} \mathrm{~N}_{3} \mathrm{O}: 205.1214\right)$. MS m/z: $205\left(\mathrm{M}^{+}, 38\right), 137$ (35), 119 (34), 85 (37), 69 (88), 56 (80), 44 (100).

1,4-Bis(phenylcarbamoyl)piperazine White solid, $\mathrm{mp}>300^{\circ} \mathrm{C}$ (lit., ${ }^{36}$, $320-321^{\circ} \mathrm{C}$ ). ${ }^{1} \mathrm{H}-\mathrm{NMR}$ (DMSO- $\left.d_{6}\right) \delta: 8.56(2 \mathrm{H}$, br s, NH), $7.45(4 \mathrm{H}$, dd, $J=8.4,0.9 \mathrm{~Hz}), 7.23(4 \mathrm{H}, \mathrm{dd}, J=8.4,7.5 \mathrm{~Hz}), 6.93(2 \mathrm{H}, \mathrm{tt}, J=7.5,0.9 \mathrm{~Hz})$, $3.48(8 \mathrm{H}, \mathrm{s})$. HR-MS $m / z: 324.1588$ (Calcd for $\mathrm{C}_{18} \mathrm{H}_{20} \mathrm{~N}_{4} \mathrm{O}_{2}: 324.1585$ ). MS m/z: $324\left(\mathrm{M}^{+}, 3.5\right), 205$ (88), 137 (59), 113 (34), 92 (50), 69 (100).

$\boldsymbol{N}$-[2-(4-1H-Imidazolyl)ethyl]- $\boldsymbol{N}^{\prime}$-phenylurea (6c) Colorless prisms (AcOEt-MeOH-ether), $\quad 179-181^{\circ} \mathrm{C} \quad$ (lit., $\left.{ }^{37)} \quad 182-184{ }^{\circ} \mathrm{C}\right) . \quad{ }^{1} \mathrm{H}-\mathrm{NMR}$ $\left(\mathrm{CD}_{3} \mathrm{OD}\right) \delta: 7.59(1 \mathrm{H}, \mathrm{d}, J=1.2 \mathrm{~Hz}), 7.31(2 \mathrm{H}, \mathrm{dd}, J=8.7,1.5 \mathrm{~Hz}), 7.22(2 \mathrm{H}$, dd, $J=8.7,7.5 \mathrm{~Hz}), 6.95(1 \mathrm{H}, \mathrm{tt}, J=7.5,1.2 \mathrm{~Hz}), 6.87(1 \mathrm{H}, \mathrm{s}), 3.45(2 \mathrm{H}, \mathrm{t}$, $J=6.9 \mathrm{~Hz}), 2.79(2 \mathrm{H}, \mathrm{t}, J=6.9 \mathrm{~Hz})$. IR $(\mathrm{KBr}) \mathrm{cm}^{-1}: 3360,1640,1554,1305$ HR-MS $m / z: 230.1155$ (Calcd for $\mathrm{C}_{12} \mathrm{H}_{14} \mathrm{~N}_{4} \mathrm{O}: 230.1167$ ). MS $m / z: 230\left(\mathrm{M}^{+}\right.$, 4), 138 (25), 119 (73), 82 (100)

Preparation of $\boldsymbol{N}$-t-Butyl- $\boldsymbol{N}^{\prime}$-(3-pyridyl)urea (7) A solution of 3aminopyridine $(376 \mathrm{mg}, 4.00 \mathrm{mmol})$ and $t$-butyl isocyanate $(1.42 \mathrm{ml}$, $12.0 \mathrm{mmol})$ in THF $(5 \mathrm{ml})$ was refluxed for $7 \mathrm{~h}$ under Ar. The reaction mixture was evaporated in vacuo, and the residue was taken up in $\mathrm{H}_{2} \mathrm{O}$ then extracted with ether. The organic layer was washed with brine, dried over $\mathrm{Na}_{2} \mathrm{SO}_{3}$ and evaporated in vacuo to give $7(212 \mathrm{mg}, 27 \%)$ as colorless needles (AcOEt-hexane), mp $141-143^{\circ} \mathrm{C}$ (lit., ${ }^{38)} \mathrm{mp} 145-147^{\circ} \mathrm{C}$ ). ${ }^{1} \mathrm{H}-\mathrm{NMR}$ $\left(\mathrm{DMSO}-d_{6}\right) \delta: 8.28(1 \mathrm{H}, \mathrm{d}, J=2.4 \mathrm{~Hz}), 8.19(1 \mathrm{H}, \mathrm{dd}, J=4.7,1.5 \mathrm{~Hz}), 8.05$ $(1 \mathrm{H}$, ddd, $J=8.4,2.4,1.5 \mathrm{~Hz}), 7.44(1 \mathrm{H}$, br s, NH), $7.21(1 \mathrm{H}, \mathrm{dd}, J=8.4$, $4.7 \mathrm{~Hz}), 5.19\left(1 \mathrm{H}\right.$, br s, NH), $1.37(9 \mathrm{H}, \mathrm{s}, t-\mathrm{Bu})$. MS m/z: $193\left(\mathrm{M}^{+}, 16\right), 136$ (6), 120 (44), 94 (98), 59 (100).

$\boldsymbol{N}$-(3-Pyridyl)- $\boldsymbol{N}^{\prime}$-(5-tropolonyl)urea (8a) Dark brown granules $(\mathrm{EtOH}), 212-213{ }^{\circ} \mathrm{C} .{ }^{1} \mathrm{H}-\mathrm{NMR}$ (DMSO-d $d_{6} \delta: 9.04(1 \mathrm{H}$, br s, NH), 9.00 $(1 \mathrm{H}$, br s, NH), $8.60(1 \mathrm{H}, \mathrm{d}, J=2.4 \mathrm{~Hz}), 8.19(1 \mathrm{H}, \mathrm{dd}, J=4.7,1.2 \mathrm{~Hz}), 7.91$ $(1 \mathrm{H}, \mathrm{ddd}, J=8.4,2.4,1.2 \mathrm{~Hz}), 7.56(2 \mathrm{H}, \mathrm{d}, J=12.6 \mathrm{~Hz}), 7.31(1 \mathrm{H}, \mathrm{dd}, J=8.4$, $4.7 \mathrm{~Hz}), 7.22(2 \mathrm{H}, \mathrm{d}, J=12.6 \mathrm{~Hz}) .{ }^{13} \mathrm{C}-\mathrm{NMR}\left(\mathrm{DMSO}-d_{6}\right) \delta: 168.7,152.5$, $143.0,140.1,139.2,135.9,127.9,125.3,124.8,123.5$. HR-MS $m / z$ 257.0803 (Calcd for $\mathrm{C}_{13} \mathrm{H}_{11} \mathrm{~N}_{3} \mathrm{O}_{3}$ : 257.0800). MS m/z: $257\left(\mathrm{M}^{+}, 16\right), 214$ (9), 163 (99), 137 (100)

$\boldsymbol{N}$-(3-Pyridyl)- $\boldsymbol{N}^{\prime}$-p-tolylurea (8b) Colorless prisms (from $\mathrm{EtOH}-\mathrm{H}_{2} \mathrm{O}$ ), mp $188-189^{\circ} \mathrm{C}$. (lit., $\left.{ }^{39)} 180{ }^{\circ} \mathrm{C}\right) .{ }^{1} \mathrm{H}-\mathrm{NMR}$ (DMSO- $\left.d_{6}\right) \delta: 8.76(1 \mathrm{H}$, brs, $\mathrm{NH}), 8.65(1 \mathrm{H}$, br s, NH), $8.57(1 \mathrm{H}, \mathrm{d}, J=2.7 \mathrm{~Hz}), 8.16(1 \mathrm{H}, \mathrm{dd}, J=4.7$, $1.2 \mathrm{~Hz}), 7.96(1 \mathrm{H}$, ddd, $J=8.6,2.7,1.2 \mathrm{~Hz}), 7.33(2 \mathrm{H}, \mathrm{d}, J=8.4 \mathrm{~Hz}), 7.29$ $(1 \mathrm{H}, \mathrm{dd}, J=8.6,4.7 \mathrm{~Hz}), 7.08(2 \mathrm{H}, \mathrm{d}, J=8.4 \mathrm{~Hz}), 2.23(3 \mathrm{H}, \mathrm{s}, \mathrm{Me}) . \mathrm{MS} m / z$ : $227\left(\mathrm{M}^{+}, 35\right), 120(20), 107$ (87), $106(80), 94$ (100).

\section{References}

1) Stephens D. N., Löschmann P. A., Lanzinger C., Wachtel H., Montgomery A. M. J., Herberg L. J., Behav. Pharmacol., 1, 521-530 (1990).

2) Lebovitz H. E., Feinglos M. N., Diabetes Care, 1, 189-198 (1978).

3) Feinglos M. N., Lebovitz H. E., Nature (London), 276, 184-185 (1978).

4) Wilhelm S. M., Carter C., Tang L., Wilkie D., McNabola A., Rong H., Chen C., Zhang X., Vincent P., McHugh M., Cao Y., Shujath J., Gawlak S., Eveleigh D., Rowley B., Liu L., Adnane L., Lynch M., Auclair D., Taylor I., Gedrich R., Voznesensky A., Riedl B., Post L. E. Bollag G., Trail P. A., Cancer Res., 64, 7099 -7109 (2004).

5) Kane R. C., Farrell A. T., Saber H., Tang S., Williams G., Jee J. M., Liang C., Booth B., Chidambaram N., Morse D., Sridhara R., Garvey P., Justice R., Pazdur R., Clin. Cancer Res., 12, 7271-7278 (2006).

6) Tixier C., Sancelme M., Bonnemoy F., Cuer A., Veschambre H., Environ. Toxicol. Chem., 20, 1381-1389 (2001).

7) Okamoto T., Shudo K., Takahashi S., Kawachi E., Isogai Y., Chem. Pharm. Bull., 29, 3748-3750 (1981).

8) Review: Shudo K., Yakugaku Zasshi, 114, 577-588 (1994).

9) Sandler S. R., Karo W., "Organic Functional Group Preparations,” Vol. II, Chap. 6, ed. by Blomquist A. T., Academic Press, New York, 1971, pp. $134-165$.

10) Review: Bigi F., Maggi R., Sartori G., Green Chem., 2, 140-148 (2000).

11) Ozaki S., Chem. Rev., 72, 457-496 (1972)

12) Overman L. E., Taylor G. F., Petty C. B., Jessup P. J., J. Org. Chem., 43, $2164-2167$ (1978).

13) Knölker H.-J., Braxmeier T., Schlechtingen G., Synlett, 1996, 502504 (1996).

14) Knölker H.-J., Braxmeier T., Schlechtingen G., Angew. Chem., Int. Ed. Engl., 34, 2497-2500 (1995).

15) Izdebski J., Pawlak D., Synthesis, 1989, 423-425 (1989).

16) Lamothe M., Perez M., Colovray-Gotteland V., Halazy S., Synlett, 1996, 507-508 (1996).

17) Matsumura Y., Satoh Y., Onomura O., Maki T., J. Org. Chem., 65, 1549-1551 (2000)

18) Gallou I., Erikosson M., Zeng X., Senanayake C., Farina V., J. Org. Chem., 70, 6960-6963 (2005).

19) Nowick J. S., Powell N. A., Nguyen T. M., Noronha G., J. Org. Chem., 57, 7364-7366 (1992).

20) Kurita K., Matsumura T., Iwakura Y., J. Org. Chem., 41, 2070-2071 (1976).

21) Majer P., Randad R. S., J. Org. Chem., 59, 1937-1938 (1994). 
22) Ito A., Muratake H., Shudo K., J. Org. Chem., 74, 1275-1281 (2009).

23) Ebisawa M., Ohta K., Kawachi E., Fukasawa H., Hashimoto Y., Kagechika H., Chem. Pharm. Bull., 49, 501-503 (2001).

24) Morita Y., Matsumura E., Okabe T., Shibata M., Sugiura M., Ohe T., Tsujibo H., Ishida N., Inamori Y., Biol. Pharm. Bull., 26, 1487-1490 (2003).

25) Zhao J., Curr. Med. Chem., 14, 2597-2621 (2007).

26) Nozoe T., Seto S., Ebine S., Ito S., J. Am. Chem. Soc., 73, 1895 (1951).

27) Nozoe T., Seto S., Proc. Jpn. Acad., 27, 188-189 (1951).

28) Furuya Y., Itoho K., Chem. Ind. (London), 1967, 359-360 (1967).

29) Ramadas K., Srinivasan N., Org. Prep. Proced. Int., 25, 600-601 (1993).

30) Yang Y., Lu S., Org. Prep. Proced. Int., 31, 559—561 (1999).
31) Mukaiyama T., Fujita Y., Bull. Chem. Soc. Jpn., 29, 54-57 (1956).

32) Ozaki S., Nagoya T., Bull. Chem. Soc. Jpn., 30, 444-449 (1957).

33) Stowell J. C., Padegimas S. J., J. Org. Chem., 39, 2448-2449 (1974).

34) Gulyaev N. D., Leonova T. V., Chimishkyan A. L., J. Org. Chem. U.S.S.R. (Engl. Transl.), 19, 1909-1913 (1983).

35) Hughes M. P., Smith B. D., J. Org. Chem., 62, 4992- 4499 (1997).

36) Rivett D. E., Wilshire J. F. K., Aust. J. Chem., 19, 869-875 (1966).

37) Battersby A. R., Nicoletti M., Staunton J., Vleggaar R., J. Chem. Soc., Perkin Trans. 1, 1980, 43-51 (1980).

38) Manley P. W., Quast U., J. Med. Chem., 35, 2327-2340 (1992).

39) Stanovnik B., Tišler M., Golob V., Hvala I., Nikolič O., J. Heterocycl. Chem., 17, 733-736 (1980). 